\title{
1 RNF8 is not required for histone-to-protamine exchange in
}

\section{2 spermiogenesis}

3

4 Hironori Abe ${ }^{1,2}$, Rajyalakshmi Meduri ${ }^{3}$, Ziwei $\mathrm{Li}^{3}$, Paul R. Andreassen ${ }^{4}$, Xin Zhiguo Li ${ }^{3 *}$, and

5 Satoshi H. Namekawa ${ }^{1,2,5^{*}}$

6

$7{ }^{1}$ Division of Reproductive Sciences, Cincinnati Children's Hospital Medical Center, Cincinnati, Ohio, 845229, USA

$9{ }^{2}$ Department of Microbiology \& Molecular Genetics, University of California, Davis, California, 95616, 10 USA

$11{ }^{3}$ Center for RNA Biology: From Genome to Therapeutics, Department of Biochemistry and Biophysics,

12 Department of Urology, University of Rochester Medical Center, Rochester, New York, 14642, USA

$13{ }^{4}$ Division of Experimental Hematology \& Cancer Biology, Cincinnati Children's Hospital Medical Center,

14 Cincinnati, Ohio, 45229, USA

$15 \quad{ }^{5}$ Lead Contact

16 *Correspondence: xin_li@urmc.rochester.edu, snamekawa@ucdavis.edu, 17

18 Keywords: Sperm, Spermiogenesis, histone-to-protamine exchange, MIWI, RNF8 


\section{Summary}

20 A member of the PIWI family of proteins, MIWI, binds to Piwi-interacting RNA (piRNA) and is essential

21 in mouse spermiogenesis. A recent study demonstrated that MIWI is an essential regulator of the histone-

22 to-protamine exchange in spermiogenesis and that this function is mediated by its binding to an ubiquitin

23 ligase, RNF8. However, here we confirm that RNF8 is not required for histone-to-protamine exchange in

24 spermiogenesis. We show that histone-to-protamine exchange takes place in Rnf8-deficient mice, while

25 RNF8 mediates ubiquitination of $\mathrm{H} 2 \mathrm{~A}$ on the sex chromosomes in meiosis, the prior stage of

26 spermatogenesis. Therefore, the infertile phenotype of MIWI mutant mice cannot be explained by a RNF8-

27 mediated mechanism in spermiogenesis. 
Introduction

To prepare fertilization, male germ cells undergo a massive nuclear transformation in which nuclear

30 histones are largely replaced with highly basic proteins, called protamines, to produce condensed sperm

31 nuclei. The mechanism underlying histone-to-protamine exchange remains largely unknown, although

32 several key aspects have been identified. Genome-wide histone acetylation represents an initial signal for

33 histone-to-protamine exchange (Goudarzi et al., 2014). Following acetylation, the histone variant H2A.L.2

34 works with transition proteins, TNP1 and TNP2, for the loading of protamines (Barral et al., 2017). A

35 histone-ubiquitin ligase RNF8 was reported to be required for genome-wide histone acetylation and

36 subsequent histone-to-protamine exchange based on the absence, genome-wide, of histone acetylation, and

37 of two protamine proteins, PRM1 and PRM2 in Rnf8-deficient (KO) mice (Lu et al., 2010). However, using

38 the same line of Rnf8-KO mice, we showed that genome-wide accumulation of histone H4K16 acetylation

39 and incorporation of PRM1 took place in Rnf8-KO elongating spermatids; the effects of RNF8 on PRM2

40 deposition in elongating spermatids was not examined at that time (Sin et al., 2012). In contrast, we did

41 confirm a role for RNF8 in mediating genome-wide ubiquitination of H2A in elongating spermatids. Thus,

42 our previous study demonstrated that RNF8-mediated ubiquitinated H2A is associated neither with H4K16

43 acetylation nor with the histone-to-protamine exchange in elongating spermatids.

Nonetheless, later studies have continued to postulate a requirement for RNF8 in the histone-toprotamine exchange. Among these, a recent study claimed that a mouse PIWI protein, MIWI, controls

47 RNF8-mediated histone-to-protamine exchange through the direct regulation of RNF8 by MIWI in 48 spermiogenesis in mice (Gou et al., 2017). PIWI proteins are evolutionarily-conserved proteins that are 49 expressed in germ cells and are associated with Piwi-interacting RNA (piRNA) for suppression of 50 retrotransposons (Juliano et al., 2011; Siomi et al., 2011). MIWI is one of the two PIWI proteins that is 51 expressed during the pachytene stage in mice ( $\mathrm{Li}$ et al., 2013) and loss of its function leads to 52 spermiogenesis arrest (Deng and Lin, 2002). Based on the finding that MIWI regulates RNF8, Gou et al. 
53 (2017) concluded that MIWI has a piRNA-independent function, but instead has a RNF8-dependent

54 function in histone-to-protamine exchange. Further, an additional study suggested that RNF8 is required

55 for histone-to-protamine exchange based on the presence of testis-specific histone H2B in sperm nuclei of

56 Rnf8-deficient mice (Guo et al., 2018). These studies have spawned a lingering view in the field that RNF8

57 is required for histone-to-protamine exchange (Wang et al., 2019; Yan, 2017).

In the present study, to clarify the function of RNF8 in histone-to-protamine exchange, we

rigorously examined whether RNF8 is involved in this process in two independent laboratories at different

61 institutions (the Namekawa and Li laboratories). Here, we demonstrate that histone-to-protamine exchange

62 takes place in Rnf8-deficient mice, confirming our previous conclusion that RNF8 is not required for

63 histone-to-protamine exchange in spermiogenesis. Instead, RNF8 has a nuclear function in the

64 ubiquitination of the sex chromosomes during meiosis and in the activation of sex chromosome-linked

65 genes in postmeiotic spermatids (Adams et al., 2018; Hasegawa et al., 2015; Sin et al., 2012). Therefore,

66 we again refute the findings of Lu et al. (2010) that RNF8 is required for histone-protamine replacement.

67 Furthermore, as a consequence, the infertility phenotype of MIWI mutant mice reported in Gou et al. (2017)

68 cannot be explained by a RNF8-mediated mechanism in spermiogenesis, challenging the main mechanistic

69 conclusion of that article.

\section{Results and Discussion}

To confirm whether RNF8 is required for histone to protamine exchange, we (the Namekawa and

73 Li laboratories) have independently examined Rnf8-deficient (KO) mice (Minter-Dykhouse et al., 2008).

74 The results were consistent between our two labs, and here we present representative data. This Rnf8-KO

75 mouse line was generated from an embryonic stem cell line, RRR260, in which the Rnf8 gene was disrupted

76 by a gene trap mutation, and the absence of RNF8 protein was previously confirmed (Santos et al., 2010).

77 This same line of Rnf8-KO mice was widely used in previous studies of the function of RNF8 in 
spermatogenesis (Adams et al., 2018; Guo et al., 2018; Hasegawa et al., 2015; Ichijima et al., 2011; Lu et

al., 2010; Lu et al., 2013; Santos et al., 2010; Sin et al., 2012; Sin et al., 2015), including the initial study

by Lu et al. (2010) which suggested a requirement for RNF8 in mediating histone-to-protamine exchange.

Since Lu et al. reported that RNF8-dependent histone ubiquitination induces H4K16 acetylation as

83 an initial step of histone removal (Lu et al., 2010), we first revisited whether H4K16 acetylation is RNF8-

dependent or not. In accordance with our previous conclusion (Sin et al., 2012), we confirmed that the

distribution of H4K16 acetylation was comparable between testicular sections of Rnf8-KO and control

86 littermate wild-type testes (Figure 1A). This result was independently confirmed by comparable

87 immunostaining signals of pan-acetylation on histone $\mathrm{H} 4$ between Rnf8-KO testes and wild-type controls

88 (Figure 1B). However, RNF8 is indeed required for H2A ubiquitination on entire nuclei of elongating

89 spermatids (Sin et al., 2012). Thus, we conclude that RNF8-dependent histone ubiquitination does not

90 induce genome-wide H4K16 acetylation in elongating spermatids.

Next, we examined the distribution of protamines (PRM1 and PRM2) on testicular sections of Rnf8-

93 KO mice. We found that both PRM1 and PRM2 were present, and the distributions were comparable

94 between Rnf8-KO testes and wild-type controls (Figures 1A-C). These results confirm our previous

95 conclusion that PRM1 incorporation is independent of RNF8 (Sin et al., 2012), and in addition now shows

96 that incorporation of two kinds of protamines (PRM1 and PRM2) takes place in spermiogenesis in Rnf8-

$97 \quad$ KO testes.

98

To further confirm RNF8-independent protamine incorporation, we prepared smear slides from

100 epididymal sperm of Rnf8-KO mice. Rnf8-KO epididymis contained sperm (Lu et al., 2010), suggesting

101 that spermiogenic progression was not abrogated in Rnf8-KO testes. Further, we found that PRM1 was

102 present in Rnf8-KO epididymal sperm, and the distribution of PRM1 was comparable between Rnf8-KO 
103 and wild-type sperm (Figure 2A). PRM2 was also present in Rnf8-KO epididymal sperm, and the

104 distribution of PRM2 was comparable between Rnf8-KO and wild-type sperm (Figure 2B). Together, these

105 results confirm that RNF8 is not required for the histone-to-protamine exchange.

A possible explanation for why the initial study by $\mathrm{Lu}$ et al. did not observe protamine incorporation

108 into sperm nuclei ( $\mathrm{Lu}$ et al., 2010) is the inability of Rnf8-KO spermatogenesis to reach the stage of

109 protamine incorporation in certain specific genetic backgrounds or environments. In support of this

110 possibility, Lu et al. showed that the late stages of spermiogenesis were abrogated in the Rnf 8 -KO testis.

111 (Lu et al., 2010). On the other hand, Rnf8-KO mice in our colony did not show abrogation of spermiogenic

112 progression in Rnf8-KO testes, enabling us to confirm that RNF8 is not required for histone-to-protamine

113 exchange. Curiously, the same Rnf8-KO mice were reported to be subfertile in another study (Guo et al.,

114 2018). While that study claimed that RNF8 is required for histone-protamine exchange, fertile Rnf8-KO

115 sperm must have underwent normal histone-protamine exchange to enable fertilization, supporting our

116 conclusion that RNF8 is not required for the histone-to-protamine exchange. These studies together suggest

117 that the phenotypic outcomes (spermiogenic progression) were different among the various studies due to

118 distinct genetic backgrounds or environments, thereby leading to conflicting conclusions about the role of

119 RNF8 in the histone-to-protamine exchange. Specifically, some factor(s) associated with a particular

120 genetic background and/or environment may have acted as a modifier of spermiogenic progression in $R n f 8$

121 KO mice.

In spite of the variable spermiogenic progression among studies, previous studies clearly agree that

124 RNF8 has a function in male meiosis prior to spermiogenesis. RNF8 has a nuclear function in the regulation

125 of sex chromosomes, and Rnf8-KO mice consistently show abrogation of histone ubiquitination on the sex

126 chromosome during meiosis, as also observed by Lu al. (2010) (Adams et al., 2018; Hasegawa et al., 2015;

127 Lu et al., 2010; Lu et al., 2013; Santos et al., 2010; Sin et al., 2012). Notably, this phenotype was observed 
in $100 \%$ of the meiotic cells we have examined. This complete phenotype may be due to a requirement for

129 the DDR pathway, which includes RNF8, in the regulation of unsynapsed sex chromosomes during meiosis

130 (Ichijima et al., 2011; Ichijima et al., 2012), and DDR-dependent coordination of meiotic progression (Abe

131 et al., 2020). Downstream of RNF8-mediated ubiquitination on the sex chromosomes, active epigenetic

132 modifications are established on meiotic sex chromosomes, and a portion of sex chromosome-linked genes

133 are activated in postmeiotic spermatids (Adams et al., 2018; Hasegawa et al., 2015; Sin et al., 2012; Sin et

134 al., 2015). Variable abnormal gene expression of sex chromosome-linked genes in Rnf8-KO testes could

135 modulate phenotypic outcomes (i.e. spermiogenic progression) in distinct genetic backgrounds or

136 environments among different studies (Guo et al., 2018; Lu et al., 2010; Sin et al., 2012). Additionally, a

137 chromatin-remodeling nuclear protein, CHD5 has been reported as another factor that controls

138 hyperacetylation of $\mathrm{H} 4$ at $\mathrm{K} 5 / 8 / 16$, histone-to-protamine exchange and chromatin condensation in sperm

139 heads_(Li et al., 2014; Zhuang et al., 2014), which shows substantial similarity with the Rnf8-KO phenotype

140 reported by Lu et al. (2010). This is an example of a factor that could potentially be affected by differences

141 in genetic background or environment and thereby account for variable phenotypes of the Rnf8-KO in

142 particular studies.

In the studies by Gou et al. (2017), which claimed that MIWI-dependent regulation of RNF8 is

145 critical for histone-to-protamine exchange, the authors did not directly examine Rnf 8 -KO mice. However,

146 the authors may have miscited the literature about the function of RNF8. We already described that RNF8

147 is not required for histone-to-protamine exchange (Sin et al., 2012), but in Gou et al. (2017), RNF8 was

148 described to be "responsible for histone-to-protamine exchange ( $\mathrm{Lu}$ et al., 2010), but not critical for

149 protamine expression (Sin et al., 2012)". Therefore, the study by Gou et al., appeared to lack the literature-

150 based foundation that RNF8 is responsible for histone-to-protamine exchange. Gou et al. described the

151 phenotype of a novel MIWI mutation of the N-terminal D-box (heterozygous germline-specific knockin of

152 the R218A/L221A mutations on a single allele of the Miwi gene, referred to as Miwi ${ }^{+/ D B}$ ) (Gou et al., 2017). 
153 The late stage of spermiogenesis was abrogated in the testes of $M i w i^{+/ D B}$ mice (Gou et al., 2017). Although

154 the similarity between $\mathrm{Miwi}^{+/ D B}$ spermiogenesis and Rnf8-KO mice in the Lu et al. (2010) study was

155 described (Gou et al., 2017), the most straightforward interpretation is that $\mathrm{Miwi}^{+/ D B}$ spermiogeneis did not

156 reach the stage of histone-to-protamine exchange, and there is no direct evidence that the phenotype of

$157 \mathrm{Miwi}^{+/ D B}$ spermiogenesis is due to the loss-of-function of RNF8. Instead the case is built upon the

158 demonstration of an interaction between MIWI and RNF8, and the fact that modulation of MIWI levels has

159 an effect on RNF8 localization to nuclei.

160

Further, Gou et al. examined RNF8 using a commercial anti-RNF8 antibody. However, the

162 antibody does not specifically recognize RNF8; We detected non-specific signals in Western blots of 163 testicular lysates and immunostaining of testicular sections using Rnf8-KO testes (Figure $1 \mathrm{C}$ and 3 ). In fact,

164 there was no evidence of a specific RNF8 signal on Western blots (Figure 3). This is a key point because

165 as noted above, the evidence for a MIWI-dependent role of RNF8 in histone-to-protamine exchange was

166 indirect and rested in significant part on a purported role for MIWI in controlling nuclear localization of

167 RNF8; potential utilization of a non-specific antibody for that experiment clearly calls the result and 168 conclusion into question. Taken together, the infertile phenotype of $M i w i^{+/ D B}$ mice reported in Gou et al.

169 (2017) cannot be explained by the RNF8-mediated mechanism in spermiogenesis, challenging the main 170 mechanistic conclusion made by Gou et al. (2017).

172 Figure Legends

173 Figure 1. RNF8 is not required for H4K16 acetylation and histone-to-protamine exchange in

174 spermiogenesis.

175 (A, B, C) Testis sections from wild-type (WT) and Rnf8-KO mice immunostained with antibodies shown 176 in the panels (H4K16ac: H4K16 acetylation; H4ac: pan-acetyl H4). Dashed squares are magnified in panels 177 to the right. Scale bars: $100 \mu \mathrm{m}$. 
Figure 2. RNF8 independent incorporation of PRM1 and PRM2 in epididymal sperm.

180 (A, B) Smear preparations from wild-type (WT) and Rnf8-KO epididymal sperm immunostained with antibodies against PRM1 or PRM2. Scale bars: $5 \mu \mathrm{m}$.

Figure 3. Failure of an anti-RNF8 antibody to specifically recognize RNF8

Western blot of wild-type (WT) and Rnf8-KO lysates from whole mouse testes probed with an anti-RNF8 antibody. $40 \mu \mathrm{g}$ protein of sample was loaded in each lane. Two independent samples for WT and Rnf8extracts in WT and Rnf8-KO lysates, and no other prominent band displayed a clear difference between these genotypes.

\section{METHODS}

\section{Mouse lines}

194 The Rnf8-KO we utilized was generated previously from the embryonic stem cell line RRR260 (Bay

195 Genomics) (Minter-Dykhouse et al., 2008) and is on C57BL/6 backgrounds. All subsequent experimental work was performed under protocol no. IACUC2018-0040 approved by the Institutional Animal Care and

197 Use Committee of Cincinnati Children's Hospital and Medical Center.

\section{Immunohistochemistry}

200 For the preparation of testis paraffin blocks, excised testes were fixed with $4 \%$ paraformaldehyde in 201 phosphate-buffered saline (PBS) containing $0.1 \%$ Triton $\mathrm{X}-100$ at $4^{\circ} \mathrm{C}$ overnight. Testes were dehydrated 202 and embedded in paraffin. For histological analyses, $6 \mu \mathrm{m}$-thick paraffin sections were deparaffinized. The 
sections were autoclaved in Target Retrieval Solution, Citrate pH 6.1 (DAKO, S-1700) or Antigen

204 Unmasking Solution, Citric Acid Based (Vector, H-3300), $121^{\circ} \mathrm{C}, 100 \mathrm{kPa}(15 \mathrm{psi})$ for $10 \mathrm{~min}$. The sections

205 were blocked with Blocking One Histo (Nacalai USA, 06349-64) at room temperature (RT) for 10 min;

206 then, the sections were incubated with primary antibodies diluted in PBS at $4^{\circ} \mathrm{C}$ overnight. The following

207 antibodies were used at the indicated dilutions [format: host anti-protein (source or company with

208 product/catalog number), dilution]: rabbit anti-H4K14ac (Millipore, 06-762), 1/200; rabbit anti-H4ac

209 (Millipore, 06-866), 1/200; mouse anti- Protamine 1 (Briar Patch Biosciences, Hup1N), 1/200; goat anti-

210 Protamine 2 (Santa Cruz, sc-23104), 1/200; rabbit anti-RNF8 antibody (Proteintech, 14112-1-AP). The

211 resulting signals were detected with appropriate secondary antibodies conjugated to Alexa 488 or 555

212 (ThermoFisher Scientific), diluted 1/1000 in PBS and incubated at RT for $1 \mathrm{~h}$. Sections were counterstained

213 with the DNA-binding chemical 40 ,6-diamidino-2-phenylindole (DAPI; Sigma, D9542-5MG) diluted to 1

$214 \mathrm{mg} / \mathrm{mL}$ in water for $5 \mathrm{~min}$, then mounted with ProLong ${ }^{\mathrm{TM}}$ Gold Antifade Mountant (Thermo Fisher

215 Scientific, P36930). Images were obtained with an A1RSi Inverted Confocal Microscope (Nikon) and

216 processed with NIS-Elements Basic Research (Nikon), Photoshop (Adobe), and Illustrator (Adobe).

\section{Smear preparations}

219 Sperm were obtained from cauda epididymides of WT and Rnf8-KO at 11 weeks of age. To make sperm

220 suspensions, first, a drop of $400 \mu \mathrm{L}$ of M2 medium (Sigma, M7167) was prepared on a $35 \mathrm{~mm}$ plastic dish

221 and it was fully covered with heavy paraffin oil (Sigma, PX0046-1); then the dish was kept on a hot plate

222 at $37^{\circ} \mathrm{C}$ until use. The obtained cauda were placed in the paraffin oil and a drop of sperm was squeezed out

223 from the cauda using a forcep and a needle. The sperm drop was transferred into the drop of M2 medium.

224 After incubation at $37^{\circ} \mathrm{C}, 5 \% \mathrm{CO} 2$ for $30 \mathrm{~min}$, sperm swimming up in M2 medium were collected using

225 pipettes. After washing two times with PBS followed by centrifugation at 3,000 $\mathrm{g}$ for 3 min at RT, sperm

226 pellets were resuspended into $200 \mu \mathrm{L}$ of PBS and sperm numbers were counted. An aliquot containing $1 \mathrm{x}$

$22710^{5}$ sperm was used to make smears and slides were stored at $-80^{\circ} \mathrm{C}$ until staining was performed. 


\section{Immunostaining on sperm}

230 We followed a previously described protocol (Balhorn et al., 2018). Mouse anti-Prm1 antibody

231 (1:500 dilution; Briar Patch Biosciences, Livermore, CA, USA; Hup1N) and mouse anti-Prm2

232 antibody (1:200 dilution; Briar Patch Biosciences, Livermore, CA, USA; Hup2B) were used.

233 Secondary antibodies conjugated with either Alexa Fluor 488 or 594 (Molecular Probes, Eugene,

234 OR, USA) were used at a dilution of 1:500.

236 Western blotting

237 Western blot experiments were replicated twice; for each experiment, all samples were run on the same gel.

238 Detunicated testis pieces obtained from matured adult WT and Rnf8-KO mice were homogenized in RIPA 239 buffer (50 mM Tris- $\mathrm{HCl} \mathrm{pH} 7.5,150 \mathrm{mM} \mathrm{NaCl}, 0.1 \%$ SDS, 1\% Triton X-100, 1\% sodium deoxycholate)

240 containing cOmplete Protease Inhibitor Cocktail (Roche, 11697498001); then, the homogenate was

241 incubated on ice for $30 \mathrm{~min}$. After DNA fragmentation by sonication and subsequent centrifugation at

$24210,000 \times g$ at $4^{\circ} \mathrm{C}$, the supernatant was transferred to a new tube before total protein concentration was

243 quantified via Bradford assays. Volumes of lysates containing $40 \mu \mathrm{g}$ proteins were separated by

244 electrophoresis on $10 \%$ SDS-PAGE gels. Then, the proteins were transferred onto a PVDF membrane

245 (EMD Millipore, IPVH00010). The membranes were blocked with StartingBlockTM T20 (TBS) Blocking

246 Buffer (ThermoFisher Scientific, 37543) at RT for 30 min before incubation with anti-RNF8 antibody

247 (Proteintech, 14112-1-AP), diluted 1/2000 in Tris-buffered saline containing 0.1\% Tween 20 detergent

248 (TBST), at $4^{\circ} \mathrm{C}$ overnight. On the next day, after washing three times in TBST, 5 min per wash, the blot

249 was incubated with VeriBlot for IP Detection Reagent (HRP) (Abcam, ab131366), diluted 1/5000 in TBST

250 at RT, for $1 \mathrm{~h}$. The blot was washed three times in TBST, 5 min per wash, before incubation in Immobilon

251 Western Chemiluminescent HRP Substrate (EMD Millipore, WBKLS0500) at RT for 1 min; then, the blot 
254 (ThermoFisher Scientific, 21059) at RT for $20 \mathrm{~min}$; then, the stripped blot was washed two times in TBST, 2555 min per wash followed by blocking with StartingBlockTM T20 (TBS) Blocking Buffer at RT for 30 min, 256 prior to incubation with anti-Lamin B1 antibody (Abcam, ab16048), diluted 1/2000 in TBST, at RT for 1

257 h. After washing the blot three times in TBST, 5 min per wash, the blot was incubated with VeriBlot for IP 258 Detection Reagent (HRP), diluted 1/5000 in TBST, at RT for $1 \mathrm{~h}$, and bands were visualized through the 259 procedures described above.

\section{Acknowledgements}

263 We thank R. Balhorn for information on Protamine antibodies and usage, and all members of the Namekawa 264 and Li laboratories for discussion and helpful comments.

267 This work was supported by the NIH Grants R01GM134731 to P.R.A., R35 GM128782 to X.Z.L., and 268 R01GM098605 to S.H.N.

\section{Author Contributions}

271 H.A. X.Z.L. and S.H.N. designed the research. H.A. and R.M. and Z.L. performed the experiments. H.A., 272 R.P.A., X.Z.L. and S.H.N. wrote the manuscript. X.Z.L. and S.H.N. supervised the investigation. All 273 authors reviewed the manuscript.

\section{Declaration of Interests}

276 The authors declare no competing interests. 


\section{References}

279 Abe, H., Alavattam, K.G., Hu, Y.C., Pang, Q., Andreassen, P.R., Hegde, R.S., and Namekawa, S.H.

280 (2020). The Initiation of Meiotic Sex Chromosome Inactivation Sequesters DNA Damage Signaling from

281 Autosomes in Mouse Spermatogenesis. Current biology : CB 30, 408-420.e405.

282 Adams, S.R., Maezawa, S., Alavattam, K.G., Abe, H., Sakashita, A., Shroder, M., Broering, T.J., Sroga

283 Rios, J., Thomas, M.A., Lin, X., et al. (2018). RNF8 and SCML2 cooperate to regulate ubiquitination and

284 H3K27 acetylation for escape gene activation on the sex chromosomes. PLoS genetics 14, e1007233.

285 Balhorn, R., Steger, K., Bergmann, M., Schuppe, H.C., Neuhauser, S., and Balhorn, M.C. (2018). New

286 monoclonal antibodies specific for mammalian protamines P1 and P2. Syst Biol Reprod Med 64, 424-

287447.

288 Barral, S., Morozumi, Y., Tanaka, H., Montellier, E., Govin, J., de Dieuleveult, M., Charbonnier, G.,

289 Coute, Y., Puthier, D., Buchou, T., et al. (2017). Histone Variant H2A.L.2 Guides Transition Protein-

290 Dependent Protamine Assembly in Male Germ Cells. Molecular cell 66, 89-101.e108.

291 Deng, W., and Lin, H. (2002). miwi, a murine homolog of piwi, encodes a cytoplasmic protein essential

292 for spermatogenesis. Developmental cell 2, 819-830.

293 Gou, L.T., Kang, J.Y., Dai, P., Wang, X., Li, F., Zhao, S., Zhang, M., Hua, M.M., Lu, Y., Zhu, Y., et al.

294 (2017). Ubiquitination-Deficient Mutations in Human Piwi Cause Male Infertility by Impairing Histone-

295 to-Protamine Exchange during Spermiogenesis. Cell 169, 1090-1104.e1013.

296 Goudarzi, A., Shiota, H., Rousseaux, S., and Khochbin, S. (2014). Genome-scale acetylation-dependent

297 histone eviction during spermatogenesis. J Mol Biol 426, 3342-3349.

298 Guo, Y., Song, Y., Guo, Z., Hu, M., Liu, B., Duan, H., Wang, L., Yuan, T., and Wang, D. (2018).

299 Function of RAD6B and RNF8 in spermatogenesis. Cell Cycle 17, 162-173. 
Hasegawa, K., Sin, H.S., Maezawa, S., Broering, T.J., Kartashov, A.V., Alavattam, K.G., Ichijima, Y.,

Zhang, F., Bacon, W.C., Greis, K.D., et al. (2015). SCML2 Establishes the Male Germline Epigenome

through Regulation of Histone H2A Ubiquitination. Developmental cell 32, 574-588.

Ichijima, Y., Ichijima, M., Lou, Z., Nussenzweig, A., Camerini-Otero, R.D., Chen, J., Andreassen, P.R., germ cells. Genes \& development 25, 959-971.

Ichijima, Y., Sin, H.S., and Namekawa, S.H. (2012). Sex chromosome inactivation in germ cells: emerging roles of DNA damage response pathways. Cellular and molecular life sciences : CMLS 69, $2559-2572$.

Juliano, C., Wang, J., and Lin, H. (2011). Uniting germline and stem cells: the function of Piwi proteins and the piRNA pathway in diverse organisms. Annual review of genetics 45, 447-469.

312 Chd5 orchestrates chromatin remodelling during sperm development. Nat Commun 5, 3812.

313 Li, X.Z., Roy, C.K., Dong, X., Bolcun-Filas, E., Wang, J., Han, B.W., Xu, J., Moore, M.J., Schimenti,

314 J.C., Weng, Z., et al. (2013). An ancient transcription factor initiates the burst of piRNA production

315 during early meiosis in mouse testes. Molecular cell 50, 67-81.

316 Lu, L.Y., Wu, J., Ye, L., Gavrilina, G.B., Saunders, T.L., and Yu, X. (2010). RNF8-dependent histone

317 modifications regulate nucleosome removal during spermatogenesis. Developmental cell 18, 371-384.

318 Lu, L.Y., Xiong, Y., Kuang, H., Korakavi, G., and Yu, X. (2013). Regulation of the DNA damage

319 response on male meiotic sex chromosomes. Nat Commun 4, 2105.

320 Minter-Dykhouse, K., Ward, I., Huen, M.S., Chen, J., and Lou, Z. (2008). Distinct versus overlapping 321 functions of MDC1 and 53BP1 in DNA damage response and tumorigenesis. The Journal of cell biology $322 \quad 181,727-735$.

323 Santos, M.A., Huen, M.S., Jankovic, M., Chen, H.T., Lopez-Contreras, A.J., Klein, I.A., Wong, N., 324 Barbancho, J.L., Fernandez-Capetillo, O., Nussenzweig, M.C., et al. (2010). Class switching and meiotic 
325 defects in mice lacking the E3 ubiquitin ligase RNF8. The Journal of experimental medicine 207, 973 -

326981.

327 Sin, H.S., Barski, A., Zhang, F., Kartashov, A.V., Nussenzweig, A., Chen, J., Andreassen, P.R., and

328 Namekawa, S.H. (2012). RNF8 regulates active epigenetic modifications and escape gene activation from

329 inactive sex chromosomes in post-meiotic spermatids. Genes \& development 26, 2737-2748.

330 Sin, H.S., Kartashov, A.V., Hasegawa, K., Barski, A., and Namekawa, S.H. (2015). Poised chromatin and

331 bivalent domains facilitate the mitosis-to-meiosis transition in the male germline. BMC biology 13, 53.

332 Siomi, M.C., Sato, K., Pezic, D., and Aravin, A.A. (2011). PIWI-interacting small RNAs: the vanguard of

333 genome defence. Nature reviews Molecular cell biology 12, 246-258.

334 Wang, X., Kang, J.Y., Wei, L., Yang, X., Sun, H., Yang, S., Lu, L., Yan, M., Bai, M., Chen, Y., et al.

335 (2019). PHF7 is a novel histone H2A E3 ligase prior to histone-to-protamine exchange during

336 spermiogenesis. Development (Cambridge, England) 146.

337 Yan, W.E.-I.-C.B.O.R. (2017). piRNA-independent PIWI function in spermatogenesis and male fertility.

338 Biol Reprod 96, 1121-1123.

339 Zhuang, T., Hess, R.A., Kolla, V., Higashi, M., Raabe, T.D., and Brodeur, G.M. (2014). CHD5 is

340 required for spermiogenesis and chromatin condensation. Mech Dev 131, 35-46. 
bioRxiv preprint doi: https://doi.org/10.1101/2020.12.05.413005; this version posted December 6, 2020. The copyright holder for this preprint (which was not certified by peer review) is the author/funder, who has granted bioRxiv a license to display the preprint in perpetuity. It is made available under aCC-BY-NC-ND 4.0 International license.

Abe_Figure 1
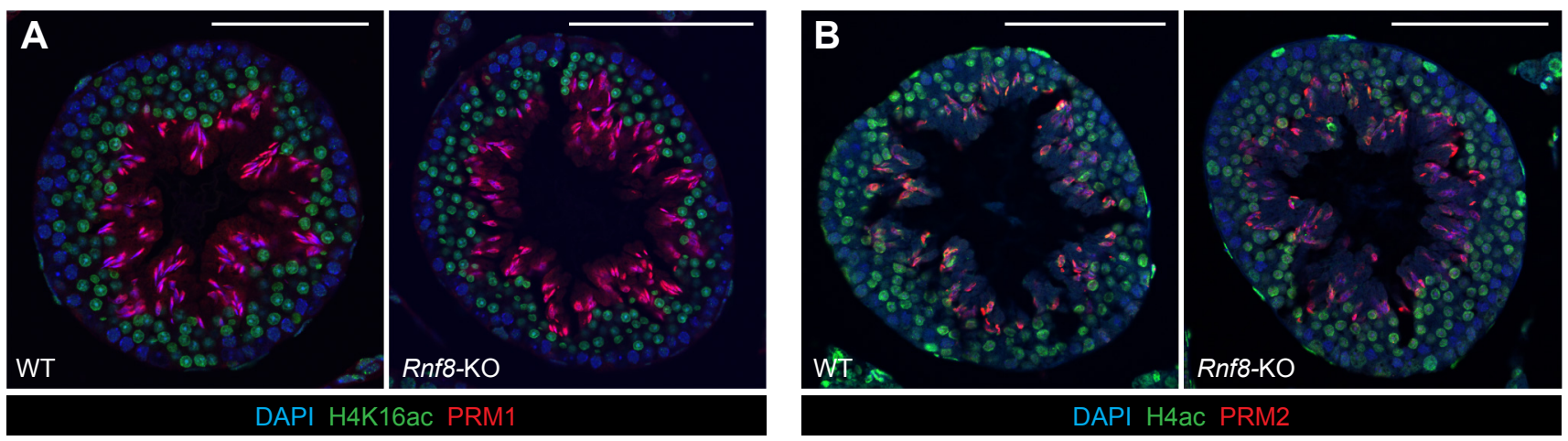

\section{C}

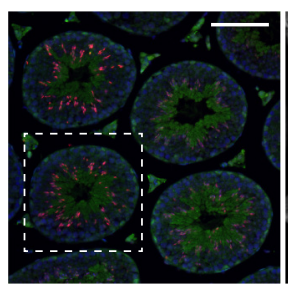

DAPI RNF8 PRM2
WT

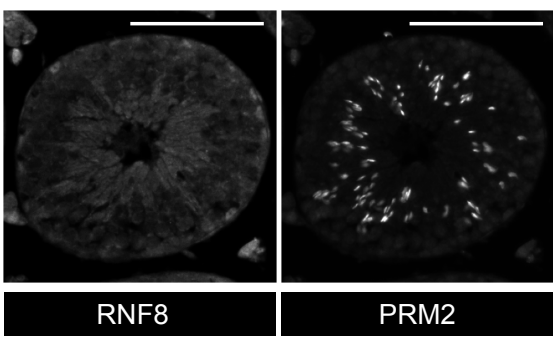

Rnf8-KO

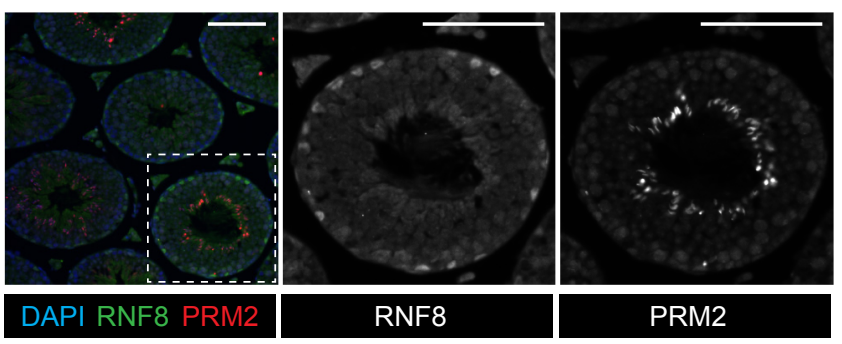


bioRxiv preprint doi: https://doi.org/10.1101/2020.12.05.413005; this version posted December 6, 2020. The copyright holder for this preprint (which was not certified by peer review) is the author/funder, who has granted bioRxiv a license to display the preprint in perpetuity. It is made available under aCC-BY-NC-ND 4.0 International license.

\section{Abe_Figure 2}
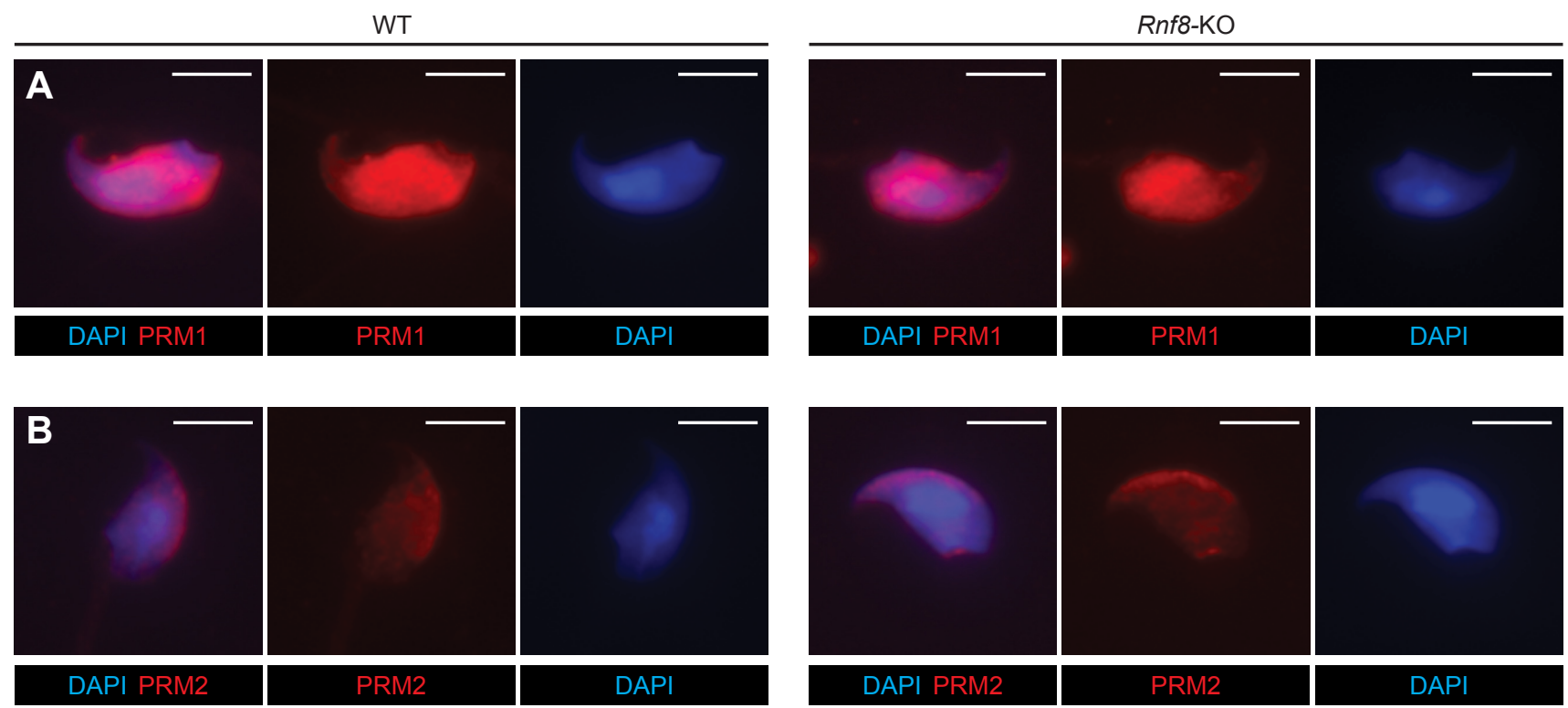
bioRxiv preprint doi: https://doi.org/10.1101/2020.12.05.413005; this version posted December 6, 2020. The copyright holder for this preprint (which was not certified by peer review) is the author/funder, who has granted bioRxiv a license to display the preprint in perpetuity. It is made available under aCC-BY-NC-ND 4.0 International license.

Abe_Figure 3

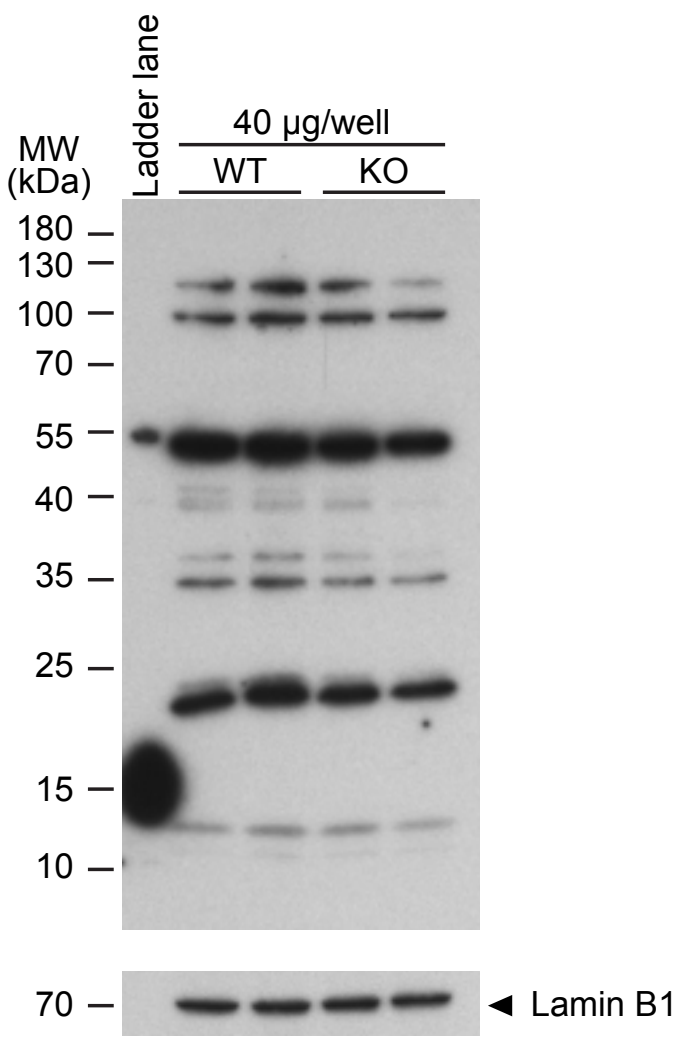

